\title{
Fusion dual-tracer SPECT-based hepatic dosimetry predicts outcome after radioembolization for a wide range of tumour cell types
}

\author{
Marnix G. E. H. Lam ${ }^{1,2}$ • Arjun Banerjee ${ }^{1}$ Michael L. Goris ${ }^{3}$. \\ Andrei H. Iagaru ${ }^{3} \cdot$ Erik S. Mittra $^{3} \cdot$ John D. Louie $^{1} \cdot$ Daniel Y. Sze $^{1}$
}

Received: 25 November 2014 / Accepted: 12 March 2015 / Published online: 28 April 2015

(C) The Author(s) 2015. This article is published with open access at Springerlink.com

\begin{abstract}
Purpose Fusion dual-tracer SPECT imaging enables physiological rather than morphological voxel-based partitioning and dosimetry for ${ }^{90} \mathrm{Y}$ hepatic radioembolization (RE). We evaluated its prognostic value in a large heterogeneous cohort of patients with extensive hepatic malignancy.

Methods A total of 122 patients with primary or secondary liver malignancy (18 different cell types) underwent SPECT imaging after intraarterial injection of ${ }^{99 \mathrm{~m}} \mathrm{Tc}$ macroaggregated albumin (TcMAA) as a simulation of subsequent ${ }^{90} \mathrm{Y}$ microsphere distribution, followed by administration of an excess of intravenous ${ }^{99 \mathrm{~m}} \mathrm{Tc}$-labelled sulphur colloid (TcSC) as a biomarker for functional liver, and a second SPECT scan. TcMAA distribution was used to estimate ${ }^{90} \mathrm{Y}$ radiation absorbed dose in tumour $\left(D_{\mathrm{T}}\right)$ and in functional liver. Laboratory and clinical follow-up were recorded for 12 weeks after RE, and radiographic responses according to $(\mathrm{m})$ RECIST were evaluated at 3 and 6 months. Dose-response relationships were determined for efficacy and toxicity.
\end{abstract}

Electronic supplementary material The online version of this article (doi:10.1007/s00259-015-3048-z) contains supplementary material, which is available to authorized users.

Marnix G. E. H. Lam

m.lam@umcutrecht.nl

1 Division of Interventional Radiology, Stanford University, Stanford, CA, USA

2 Department of Radiology and Nuclear Medicine, University Medical Center Utrecht, Heidelberglaan 100, 3584, CX Utrecht, The Netherlands

3 Division of Nuclear Medicine and Molecular Imaging, Stanford University, Stanford, CA, USA
Results Patients were treated with a median of $1.73 \mathrm{GBq}$ activity of resin microspheres ( 98 patients) or glass microspheres (24 patients), in a whole-liver approach (97 patients) or a lobar approach (25 patients). The objective response rate was $41 \%$ at 3 months and $48 \%$ at 6 months. Response was correlated with $D_{\mathrm{T}}(P<0.01)$. Median overall survival was 10.1 months (95\% confidence interval $7.4-12.8$ months). Responders lived for 36.0 months compared to 8.7 months for nonresponders $(P<0.01)$. Stratified for tumour cell type, $D_{\mathrm{T}}$ was independently associated with survival $(P<0.01)$. Absorbed dose in functional liver was correlated with toxicity grade change $(P<0.05)$ and RE-induced liver disease $(P<0.05)$. Conclusion Fusion dual-tracer SPECT imaging offers a physiology-based functional imaging tool to predict efficacy and toxicity of RE. This technique can be refined to define dosing thresholds for specific tumour types and treatments, but appears generally predictive even in a heterogeneous cohort.

Keywords Radioembolization · Treatment planning · Functional imaging $\cdot$ Partition model $\cdot$ SPECT

\section{Introduction}

Treatment planning is critical to the success of ${ }^{90} \mathrm{Y}$ hepatic radioembolization (RE) [1]. Safety and efficacy are contingent upon inhomogeneous intrahepatic distribution of radioactive microspheres to achieve tumoricidal doses without serious hepatic injury. Current standard methods for activity calculation for both resin and glass microspheres were largely validated on empirical grounds [2-8]. Although these early dose ranging studies were sufficient to show promising results and an acceptable toxicity profile, imperfect response rates and 
occasional severe toxicities continue to drive investigators towards optimization of RE treatment planning [9].

Recent proposals to improve treatment planning are based on morphological partition modelling using CT or MRI to delineate the anatomical borders of tumours within the liver volume, combined with SPECT imaging to calculate the activity distribution within these target volumes $[10,11]$. These methods incorporate dosimetric parameters such as tumour and normal liver absorbed doses in the activity calculations, and seem to be feasible and accurate in patients with a limited number of clearly demarcated tumours, mostly hepatocellular carcinomas (HCC) [12]. However, in the presence of diffuse, infiltrative $\mathrm{HCC}$ or myriad heterogeneous metastases, anatomical partition modelling is prone to significant error [1]. These partition methods are therefore difficult to standardize in clinical practice.

We proposed a new partition method using physiological parameters only: a segmentation tool based on a dual-tracer SPECT technique, combining ${ }^{99 \mathrm{~m}} \mathrm{Tc}$ macroaggregated albumin (TcMAA) SPECT for simulation of ${ }^{90} \mathrm{Y}$ activity distribution, and ${ }^{99 \mathrm{~m}} \mathrm{Tc}$-sulphur colloid (TcSC) SPECT for identifying functional liver parenchyma [13]. This method obviates the need to delineate the different compartments by anatomical imaging and is automated, fast, and objective. In a homogeneous cohort of 25 patients with metastatic colorectal carcinoma (mCRC), we found that the calculated absorbed doses to the tumour compartment $\left(D_{\mathrm{T}}\right)$ and to the functional liver compartment $\left(D_{\text {FL-TOT }}\right)$ are significantly correlated with efficacy and toxicity. In the present study, we broadened validation to a large heterogeneous cohort of patients with many different tumour cell types of different anatomical and vascular characteristics.

\section{Materials and methods}

\section{Patient selection}

Between June 2004 and September 2011, 247 consecutive patients underwent intraarterial TcMAA imaging as part of their RE preparatory work-up. Of these, 184 underwent intravenous TcSC imaging. In 38 patients, mismatch in injection positions of TcMAA and ${ }^{90} \mathrm{Y}$ jeopardized the accuracy of distribution simulation and these patients were excluded. In addition, 24 patients who received treatment in two staged sessions were excluded because pretreatment TcMAA was injected nonstaged and nonselectively. A total of 122 patients (68 men, 54 women; median age 62 years, range 25 - 92 years) were included in the analysis, including 25 previously studied patients [13]. Baseline, procedural, and follow-up data were collected prospectively as standard of care, and retrospectively analysed for this study. Data were handled in accordance with the Health Insurance Portability and Accountability Act. The institutional review board approved the study. Table 1 summarizes the patient characteristics.

\section{Radioembolization}

Activity calculations and treatments were performed according to international consensus guidelines [14-16]. Resin microspheres (SIR-Spheres; SIRTex Medical Ltd., North Sydney, Australia) were used to treat 98 patients $(80.3 \%)$. The prescribed activity was calculated based on body surface area (BSA) and estimated tumour liver involvement (LI; median $25 \%$, range $5-70 \%$ ), where prescribed activity $(\mathrm{GBq})=\mathrm{BSA}$ $\left(\mathrm{m}^{2}\right)-0.2+\mathrm{LI}$ [12]. Glass microspheres (TheraSphere; BTG, Inc., Farnham, UK) were used to treat 24 patients, applying a medical internal radiation dosimetry (MIRD) method to prescribe a desired target territory absorbed dose of $90-120$ Gy [17]. Hepatopulmonary shunting was compensated for by the recommended activity adjustments $[17,12]$.

Clinical and laboratory follow-up were performed 2, 4, 8 and 12 weeks after treatment, and at intervals prescribed by the medical oncologist thereafter. Toxicity was graded according to National Cancer Institute common toxicity criteria for adverse events (NCI-CTCAE v4.03). Follow-up imaging replicating the pretreatment modality was used for response analysis according to modified Response Evaluation Criteria in Solid Tumors (mRECIST) [18] for HCC and RECIST 1.1 for other tumour types, with a focus on liver response only [19]. One blinded reviewer performed over-reads of all clinical interpretations.

\section{Imaging procedures}

As described previously [13], routine SPECT was performed after intraarterial administration of a small TcMAA dose (37 MBq). Without moving the patient, an excess of TcSC (185 MBq) was administered intravenously, and SPECT was repeated after a 5-min delay. SPECT data were acquired on a dual-head Infinia Hawkeye 4 gamma camera (GE Healthcare, Waukesha, WI), with a $64 \times 64$ matrix (voxel size $0.884 \mathrm{~mm}^{3}$ ), $130-150 \mathrm{keV}$ energy window, low-energy high-resolution collimator and 120 projections (15 s per projection) over a $360^{\circ}$ full circular orbit.

\section{Image processing and analysis}

Data were reconstructed using filtered back projection and a Butterworth postreconstruction filter ( $\mathrm{Fc} 0.23$; order 6), using Segami software (Segami, Columbia, MD). From the TcMAA SPECT images (Fig. 1a), a three-dimensional tumour map (Fig. 1b) was generated by applying a threshold including all voxels with $10 \%$ or more of the maximum TcMAA per voxel, using software programmed in IDL 6.1 (Exelis, Inc., McLean, VA). This threshold was chosen after comparison between 5-30 \%. Corrected TcSC images (Fig. 1c) were then 
Table 1 Demographics, baseline characteristics and oncological histories of the cohort

\begin{tabular}{|c|c|}
\hline Characteristic & Value \\
\hline Sex (male/female), $n$ & $68 / 54$ \\
\hline Age (years), median (range) & $62(25-92)$ \\
\hline \multicolumn{2}{|l|}{ Tumour cell type, $n(\%)$} \\
\hline Hepatocellular carcinoma & $26(21.3)$ \\
\hline Cholangiocarcinoma & $18(14.7)$ \\
\hline Metastatic neuroendocrine carcinoma & $20(16.4)$ \\
\hline Metastatic colorectal carcinoma & $29(23.8)$ \\
\hline Other $^{\mathrm{a}}$ & $29(23.8)$ \\
\hline \multicolumn{2}{|l|}{ Previous systemic treatment, $n(\%)$} \\
\hline Cytotoxic chemotherapy & $74(60.7)$ \\
\hline Bevacizumab & $36(29.5)$ \\
\hline Sorafenib & $12(9.8)$ \\
\hline Anti-EGFR agents & $18(14.8)$ \\
\hline \multicolumn{2}{|l|}{ Previous liver-directed treatment, $n(\%)$} \\
\hline Transarterial (chemo)embolization & $27(22.1)$ \\
\hline Partial liver resection & $26(21.3)$ \\
\hline Radiofrequency ablation & $18(14.8)$ \\
\hline External beam radiation therapy ${ }^{\mathrm{b}}$ & $8(6.6)$ \\
\hline Hepatic radioembolization & $7(5.7)$ \\
\hline \multicolumn{2}{|l|}{ ECOG performance status, $n(\%)$} \\
\hline 0 & $54(44.3)$ \\
\hline 1 & $58(47.5)$ \\
\hline 2 & $10(8.2)$ \\
\hline Extrahepatic disease, $n(\%)$ & $61(50)$ \\
\hline Lung & $37(30.3)$ \\
\hline Lymph nodes & $16(13.1)$ \\
\hline Bone & $10(8.2)$ \\
\hline Estimated liver tumour involvement (\%), median (range) & $25(5-70)$ \\
\hline Maximum tumour diameter (mm), median (range) & $52(15-133)$ \\
\hline Liver cirrhosis, $n(\%)$ & $22(18)$ \\
\hline \multicolumn{2}{|l|}{ Microspheres, $n(\%)$} \\
\hline Resin & $98(80.3)$ \\
\hline Glass & $24(19.7)$ \\
\hline \multicolumn{2}{|l|}{ Treatment, $n(\%)$} \\
\hline Whole liver & $97(79.5)$ \\
\hline Lobar & $25(20.5)$ \\
\hline Administered activity (GBq), median (range) & $1.73(0.43-6.21)$ \\
\hline
\end{tabular}

EGFR epidermal growth factor receptor

${ }^{a}$ Includes sarcoma (five patients), melanoma (four), renal cell carcinoma (three), pancreatic adenocarcinoma (three), oesophageal carcinoma (three), ovarian carcinoma (one), urothelial carcinoma (one), small-cell lung carcinoma (one), lymphoma (one), cervical carcinoma (one), thymic carcinoma (two), breast carcinoma (two), ampullary carcinoma (one), and gastronintestinal junction carcinoma (one)

${ }^{\mathrm{b}}$ The mean radiation absorbed dose to the liver was determined by dose-volume histogram analysis. All mean liver doses $>1$ Gy were included calculated by subtracting TcMAA images (Fig. 1a) from the TcSC images. A map of functional liver (Fig. 1d) was produced by applying a $10 \%$ threshold to the corrected TcSC images. Fusing the TcMAA and TcSC maps (Fig. 1e) resulted in hepatic segmentation into four compartments: (1) the unirradiated functional liver compartment ( $V_{\mathrm{FL}-\mathrm{UN}}, \mathrm{TcSC}$ positive only; Fig. 1f); (2) the tumour compartment $\left(V_{\mathrm{T}}\right.$, TcMAA-positive only; Fig. 1g); (3) the overlap area, the irradiated functional liver compartment $\left(V_{\mathrm{FL}-\mathrm{IR}}\right.$, both TcSC- and TcMAA-positive; Fig. 1h) which typically represented the 
Fig. 1 A threshold applied to the TcMAA SPECT image (a) defines the MAA-positive volume (b), and to the ${ }^{99 \mathrm{~m}} \mathrm{Tc}$ sulphur colloid (SC) SPECT (c) defines the SC-positive volume (d). Coregistration of the two SPECT scans results in four compartments: $\mathbf{f}$ unirradiated functional liver $\left(V_{\mathrm{FL}-\mathrm{UN}}\right)$, MAAnegative, SC-positive (blue), $\mathbf{g}$ tumour $\left(V_{\mathrm{T}}\right)$, MAA-positive SCnegative (red), $\mathbf{h}$ irradiated functional liver $\left(V_{\mathrm{FL}-\mathrm{IR}}\right)$, MAApositive SC-positive (purple), and tumour necrosis $\left(V_{\mathrm{NULL}}\right)$, MAAnegative SC-negative (black)
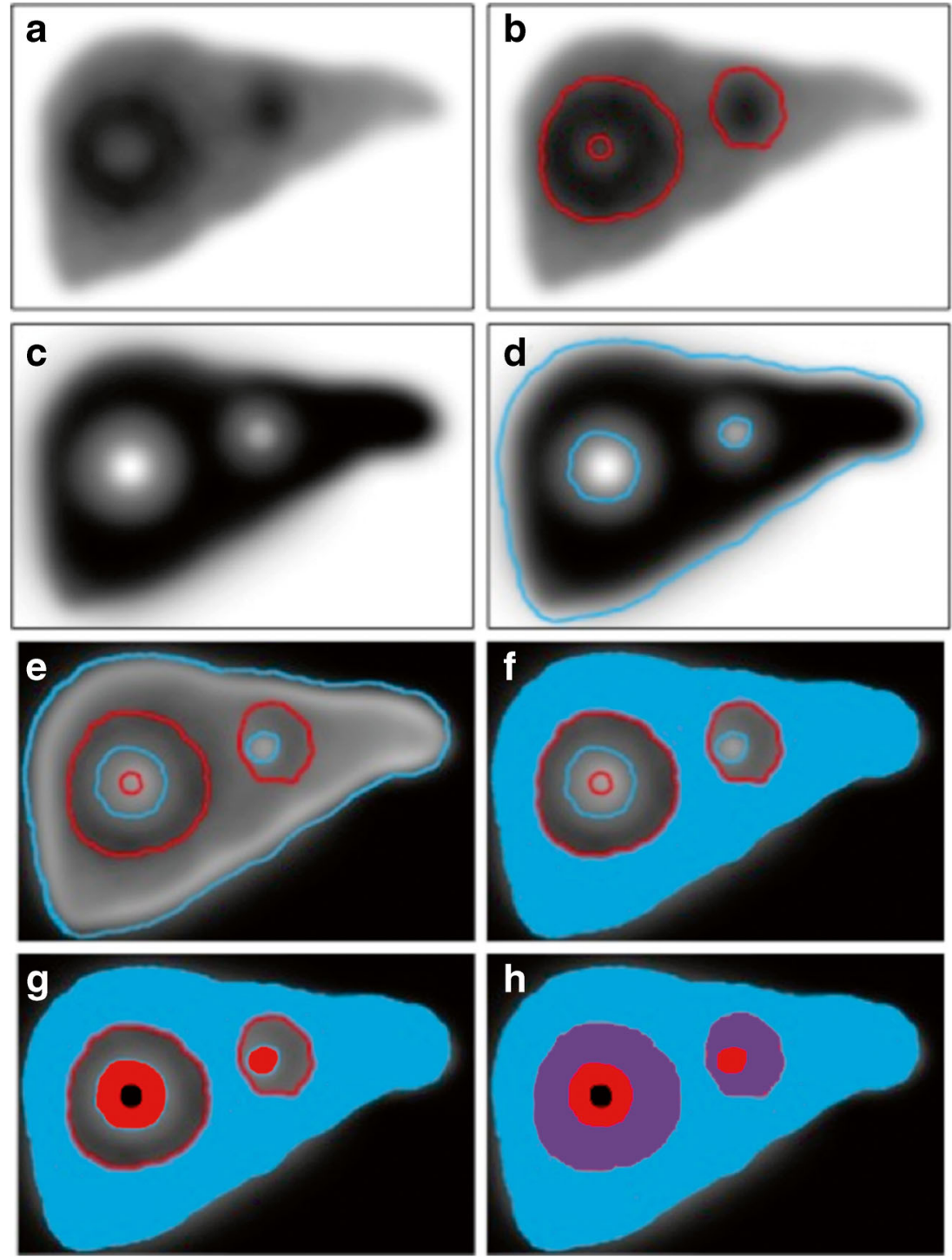

marginal zone that included hypervascular rims; and (4) the null compartment ( $V_{\mathrm{NULL}}$, both TcSC- and TcMAA-negative) which included central necrosis, major vascular structures, cysts, etc. The total functional liver compartment or $V_{\text {FL-TOT }}$ was defined as $V_{\text {FL-IR }}$ plus $V_{\text {FL-UN }}$, and the total liver volume $V_{\text {TOTAL LIVER }}$ was calculated as $V_{\mathrm{T}}$ plus $V_{\mathrm{FL}-\mathrm{TOT}}$. The process of fusion of the TcSC and TcMAA images to define the four liver compartments is shown in Fig. 2.

Voxel counts were converted into volumes, and deposited TcMAA activities were converted into compartment absorbed doses using the MIRD formula [20]:

Compartment absorbed dose $=($ compartment TcMAA activity/total TcMAA activity) $\times$ administered activity $(\mathrm{GBq}) \times 1.029^{-1}(\mathrm{~kg} / \mathrm{L}) \times$ volume $^{-1}(\mathrm{~L}) \times 50$

where 'compartment TcMAA activity' is the measured TcMAA activity in a compartment, 'total TcMAA activity' is the total TcMAA activity in the liver, $1.029 \mathrm{~kg} / \mathrm{L}$ is the estimated specific density of hepatic tissue, 'volume' is the calculated volume of the compartment (using a $10 \%$ threshold as described above), and ' 50 ' is the conversion factor for ${ }^{90} \mathrm{Y}$ from $\mathrm{GBq} / \mathrm{kg}$ to Gy. Below the $10 \%$ threshold, calculated $D_{\mathrm{FL}-\mathrm{UN}}$ to the unirradiated functional liver was simplified as zero. Calculated absorbed dose in the other three compartments $\left(D_{\mathrm{T}}, D_{\mathrm{FL}}\right.$ тот, $\left.D_{\text {FL-IR }}\right)$, volumes of each compartment ( $V_{\mathrm{T}}, V_{\mathrm{FL}-\mathrm{TOT}}, V_{\mathrm{FL}-}$ UN and $\left.V_{\text {FL-IR }}\right)$, ratios between parameters, and fractional volumes were evaluated for dose-response relationships with regard to efficacy and toxicity (Supplementary Tables 1 and 2).

\section{Statistical analysis}

A commercial software package was used for statistical analysis (SPSS for Windows, version 19.0; SPSS Inc, Chicago, IL). The normality of the distributions of continuous variables was tested using the Kolmogorov-Smirnov test. Nonparametric tests were used to compare groups (chi-squared test for categorical variables, Mann-Whitney or Kruskal-Wallis one- 
Fig. 2 Functional liver tissue defined by TcSC SPECT images in three orientations (axial, sagittal, coronal, a-c), simulated ${ }^{90} \mathrm{Y}$ distribution defined by TcMAA SPECT images in three orientations $(\mathbf{d}-\mathbf{f})$, and the coregistered fused SPECT images in three orientations $(\mathbf{g}-\mathbf{i})$, resulting in the definition of four different compartments: tumour (red), tumour necrosis (black), irradiated functional liver (purple), and unirradiated functional liver (blue)

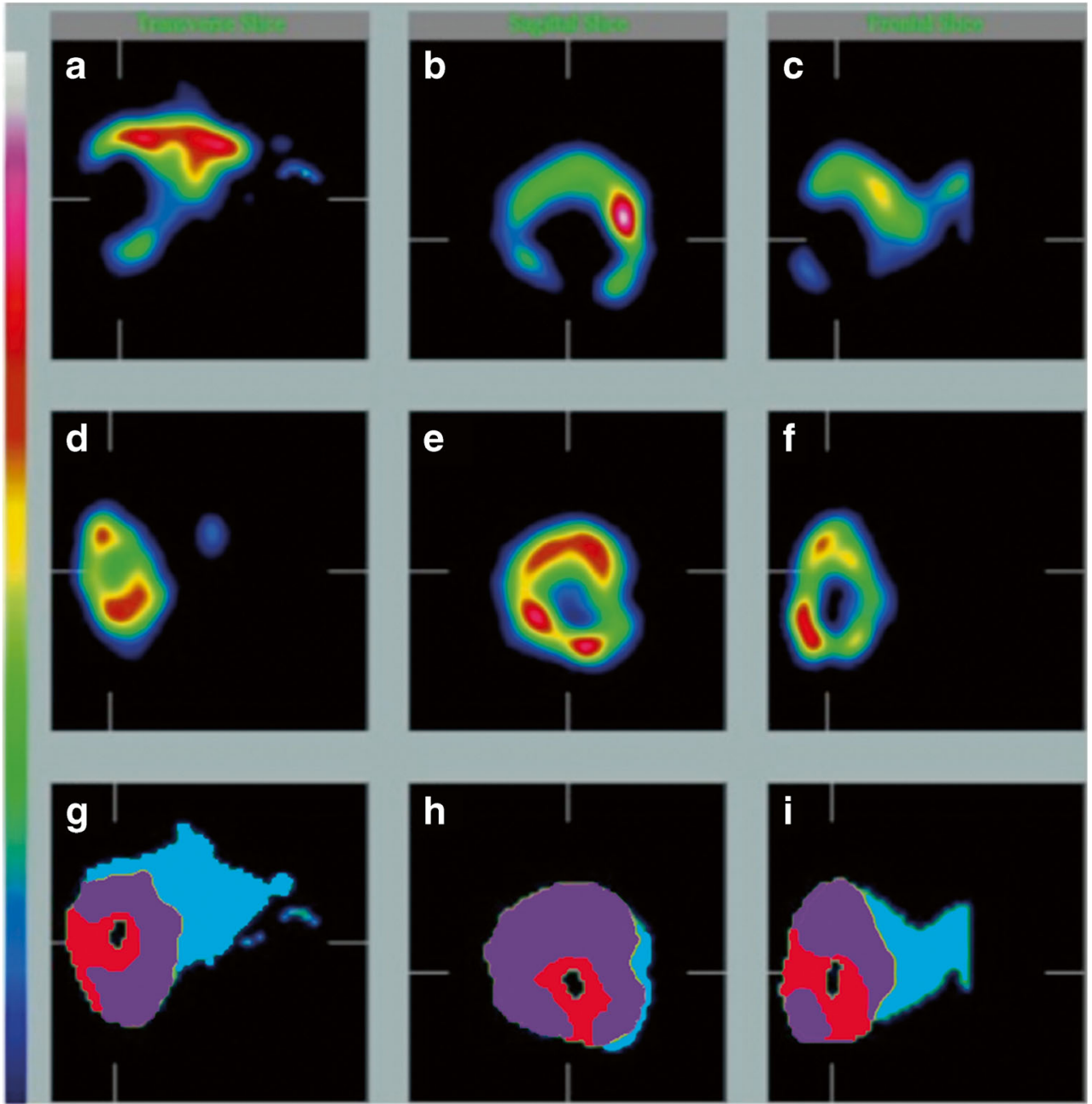

way ANOVA for continuous variables). Survival was evaluated using Kaplan-Meier curves. Stratification was performed for tumour cell type with the log rank test for comparison pooled over the strata. Multivariate survival analysis was performed with a Cox proportional hazards model using the conditional step forward method (stepwise probability: entry 0.05 , removal 0.10 ). A $P$ value $<0.05$ was considered statistically significant.

\section{Results}

The 122 patients were treated with a median of $1.73 \mathrm{GBq}$ resin microspheres (98 patients) or glass microspheres (24 patients), in a whole-liver distribution (97 microspheres) or lobar distribution (25 microspheres; Table 1$)$. The majority of patients treated with glass microspheres had HCC ( 22 of 24 patients). The median administered activity of glass microspheres was $3.37 \mathrm{GBq}$ compared to $1.64 \mathrm{GBq}$ for resin microspheres $(P<0.001)$. The median tumour absorbed dose $\left(D_{\mathrm{T}}\right)$ in the total cohort was $36.3 \mathrm{~Gy}$, and the median functional liver absorbed dose $\left(D_{\text {FL-TOT }}\right)$ was 29.7 Gy (Table 2$)$. This was correlated significantly with tumour cell type $(P<0.001)$. Patients with $\mathrm{HCC}$ had a much higher median $D_{\mathrm{T}}(109.7 \mathrm{~Gy})$ and $D_{\text {FL-Tот }}(55.1 \mathrm{~Gy})$ than those with other cell types, mostly because treatment with higher activity of glass microspheres resulted in higher $D_{\mathrm{T}}(116 \mathrm{~Gy}$ versus $32.7 \mathrm{~Gy} ; P<0.001)$ and $D_{\text {FL-TOт }}(57.1$ Gy versus $27.3 \mathrm{~Gy} ; P<0.001)$. However, independent of the type of microspheres used, HCC also had a higher $D_{\mathrm{T}} / D_{\mathrm{FL}-\mathrm{TOT}}$ ratio (median $1.8 ; P=0.02$; Table 2), probably reflecting hypervascularity and focal tumours.

Of the 122 patients, 74 were evaluable for response at 3 months (18 died before follow-up imaging, 5 had inadequate baseline imaging, and 25 did not have adequate follow-up imaging), and 44 were evaluable at 6 months (48 died, 2 inadequate baseline imaging, 28 inadequate follow-up imaging). The objective response rates (complete plus partial responses) were $41 \%$ at 3 months and $48 \%$ at 6 months (Table 3). Response at 3 months was correlated only with $D_{\mathrm{T}}$ in the univariate analysis $(P=0.026)$ and the multivariate analysis $(P=0.004)$. Other significant dosimetry parameters (Supplementary Table 1) were strongly interrelated and were 
Table 2 Treatment parameters according to tumour cell type

\begin{tabular}{|c|c|c|c|c|}
\hline \multirow[t]{2}{*}{ Tumour cell type } & \multirow{2}{*}{$\begin{array}{l}\text { No. of } \\
\text { patients }\end{array}$} & \multirow{2}{*}{$\begin{array}{l}\text { Maximum tumour diameter } \\
(\mathrm{mm})\end{array}$} & \multicolumn{2}{|c|}{ Calculated absorbed doses (Gy) } \\
\hline & & & $\begin{array}{l}\text { Tumour, } \\
D_{\mathrm{T}}\end{array}$ & $\begin{array}{l}\text { Functional liver, } D_{\mathrm{FL}} \\
\text { тот }\end{array}$ \\
\hline $\begin{array}{l}\text { Hepatocellular } \\
\text { carcinoma }\end{array}$ & 26 & 56 & 109.7 & 55.1 \\
\hline Cholangiocarcinoma & 18 & 76 & 35 & 24.9 \\
\hline $\begin{array}{l}\text { Neuroendocrine } \\
\text { carcinoma }\end{array}$ & 20 & 56 & 24.2 & 23.7 \\
\hline Colorectal carcinoma & 29 & 46 & 33.3 & 27.8 \\
\hline Other $^{\mathrm{a}}$ & 29 & 44 & 33.6 & 29.8 \\
\hline All tumour types & 122 & 52 & 36.3 & 29.7 \\
\hline $\begin{array}{l}P \text { values (between } \\
\text { groups) }\end{array}$ & NA & 0.471 & $<0.001$ & $<0.001$ \\
\hline
\end{tabular}

Variables are reported as medians

${ }^{\text {a }}$ Tumour cell types in this group are listed in Table 1 footnote a

${ }^{\mathrm{b}}$ Nonparametric Kruskal-Wallis one-way ANOVA for multiple pairwise comparison thus excluded. At 6 months, only a trend was found for $D_{\mathrm{T}}$ in the univariate analysis $(P=0.069)$. At 3 months, responders had a median $D_{\mathrm{T}}$ of 60.1 Gy versus $32.7 \mathrm{~Gy}$ for nonresponders $(P=0.026)$, and at 6 months 60.5 Gy versus $29.3 \mathrm{~Gy}(P=$ $0.069)$. The median survival (from treatment) in responders at 3 months was 36.0 months, and in nonresponders was 8.7 months $(P=0.003$; stratified according to tumour cell type: $P=0.011$; Fig. 3).

The overall median survival from treatment was 10.1 months (95\% confidence interval $7.4-12.8$ months), and from diagnosis 37.7 months (95\% confidence interval $31.1-44.3$ months; Table 3 ). At the time of writing 34 patients were still alive with a median follow-up of 27.1 months. Survival from treatment
$(P<0.001)$ and survival from diagnosis $(P<0.001)$ were dependent on tumour cell type (Fig. 4). Patients with HCC were selected for treatment with RE rather than chemoembolization if they had very large tumours $(>8 \mathrm{~cm})$, infiltrative disease, macrovascular invasion, and/or had failed prior chemoembolization (Table 2), resulting in expected poor overall survival after treatment (Table 3). Of $26 \mathrm{HCC}$ patients for example, 22 had underlying liver cirrhosis with significantly worse liver function, and 5 had main portal vein thrombosis. Stratified according to tumour cell type, $D_{\mathrm{T}}$ was correlated with survival after treatment in the univariate analysis $(P=$ $0.004)$ and multivariate analysis $(P=0.004)$. Only response, tumour cell type, and $D_{\mathrm{T}}$ were correlated with survival.

Table 3 Treatment outcome according to tumour cell type

\begin{tabular}{|c|c|c|c|c|c|c|}
\hline \multirow[t]{2}{*}{ Tumour cell type } & \multicolumn{2}{|c|}{$\begin{array}{l}\text { Survival (months), median ( } 95 \% \text { confidence } \\
\text { interval) }\end{array}$} & \multicolumn{2}{|c|}{ Response, $n(\%)^{\mathrm{a}}$} & \multirow[t]{2}{*}{ Grade 3 or 4 toxicity, $n(\%)$} & \multirow[t]{2}{*}{ REILD } \\
\hline & From treatment & From diagnosis & At 3 months & At 6 months & & \\
\hline Hepatocellular carcinoma & $8(2.4-13.6)$ & $32.4(24.9-44)$ & $9 / 15(60)$ & $7 / 10(70)$ & $10 / 22(46)$ & 2 \\
\hline Cholangiocarcinoma & $5.7(2.0-9.4)$ & $21(12.6-29.4)$ & 2/11 (18) & $2 / 5(40)$ & $2 / 16(13)$ & 1 \\
\hline Neuroendocrine carcinoma & Not reached & Not reached & $8 / 14(57)$ & $6 / 13(46)$ & $1 / 19(5)$ & 0 \\
\hline Colorectal carcinoma & $10.8(6.1-15.5)$ & $37.9(30-45.8)$ & $5 / 19(26)$ & $3 / 10(30)$ & $5 / 28(18)$ & 1 \\
\hline Other $^{b}$ & $8.3(6-10.6)$ & $32.8(18.7-46.9)$ & $6 / 15(40)$ & $3 / 6(50)$ & $6 / 26(23)$ & 1 \\
\hline All tumour types & $10.1(7.4-12.8)$ & $37.7(31.1-44.3)$ & $30 / 74(41)$ & $21 / 44(48)$ & 24/111 (22) & 5 \\
\hline Evaluable patients & 122 & 122 & 74 & 44 & 111 & 111 \\
\hline$P$ values (between groups) ${ }^{\mathrm{c}}$ & $<0.001$ & $<0.001$ & 0.098 & 0.494 & 0.022 & NA \\
\hline
\end{tabular}

REILD radioembolization-induced liver disease

${ }^{\text {a }}$ Response includes complete response and partial response by RECIST or mRECIST, as described in the text

${ }^{\mathrm{b}}$ Tumour cell types in this group are listed in Table 1 footnote a

${ }^{\mathrm{c}}$ Log-rank test for survival comparison between groups; nonparametric chi-squared test for response and toxicity comparison between groups 


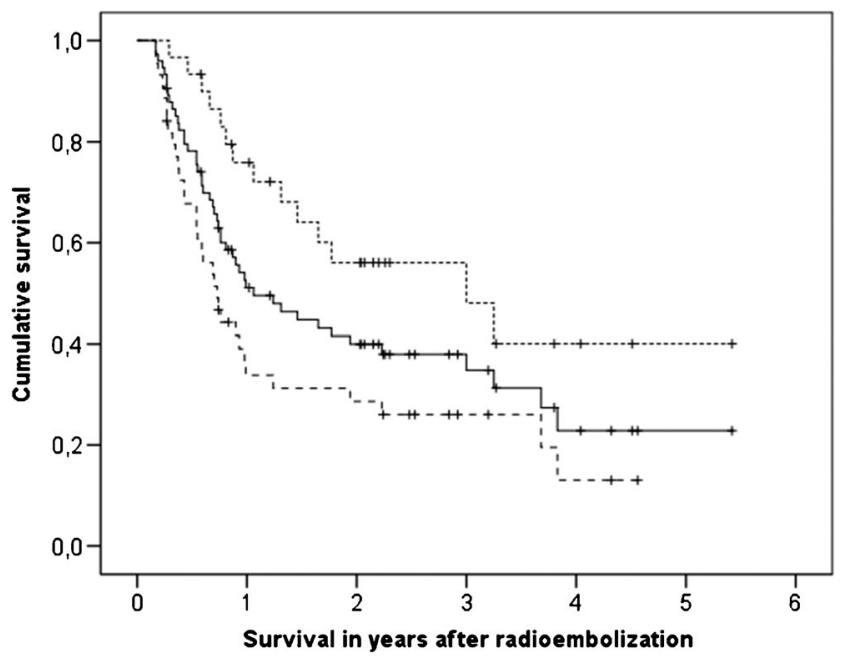

Fig. 3 Kaplan-Meier survival curves for patients with an objective response at 3 months (upper dotted line), patients without response (lower dashed line), and all patients (middle solid line). Median survival in responders was 36 months versus only 8.7 months in nonresponders $(P=0.003)$; stratified according to tumour cell type, this was still significant $(P=0.011)$

Treatment-related adverse events were usually mild, expected, and limited to grade $1 / 2$ toxicity such as nausea, abdominal discomfort and fatigue. Laboratory values changed as expected (Supplementary Fig. 1). Grade 3/4 toxicity occurred in 24 patients, most of whom had preexisting grade $1 / 2$ toxicity. Grade 4 toxicity included increases in bilirubin (two patients) and aspartate aminotransferase (AST, one patient). Absolute grade $3 / 4$ toxicity was related to tumour cell type (with a $46 \%$ incidence among those with HCC; $P=0.022)$, liver cirrhosis $(P=0.002)$, baseline AST $(P=0.002)$, and alanine aminotransferase (ALT, $P=0.004)$. However, $D_{\mathrm{FL}-\text { TOT }}$ was the strongest $(P=0.010)$ and most comprehensive dosimetry parameter associated with an increase in toxicity grade (Supplementary Table 2 and Fig. 5). Other parameters associated

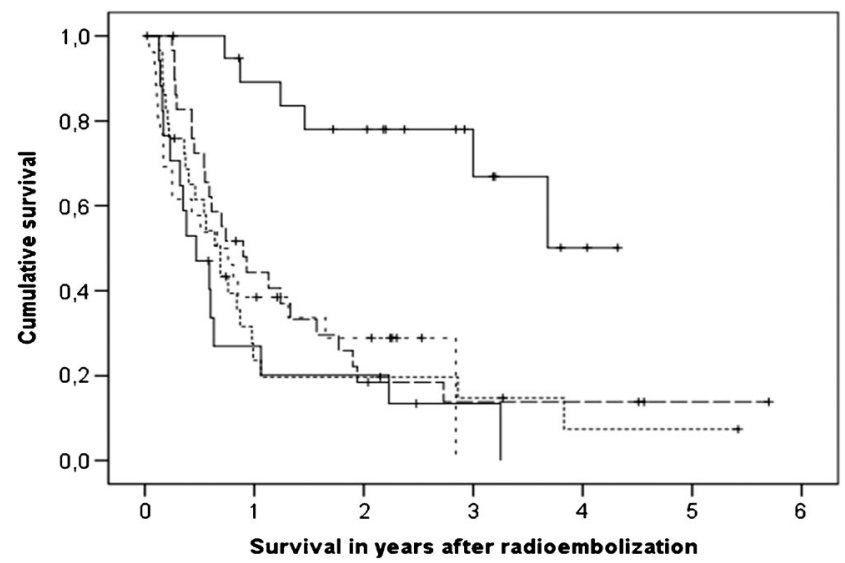

Fig. 4 Kaplan-Meier survival curves for patients with hepatocellular carcinoma (short dashes), cholangiocarcinoma (lower solid line), metastatic neuroendocrine carcinoma (upper solid line), metastatic colorectal carcinoma (long dashes), and miscellaneous metastatic malignancies (dotted line)

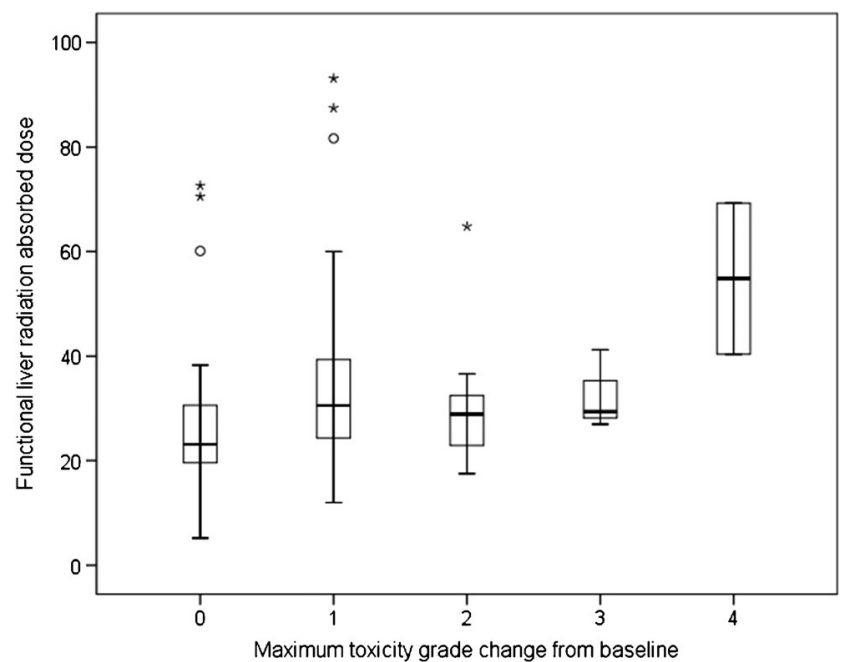

Fig. 5 Maximum change in toxicity grade from baseline during followup was significantly associated with the radiation absorbed dose to functional liver tissue $\left(D_{\mathrm{FL}-\mathrm{TOT}}\right)$. Medians, and first and third quartiles are indicated by the boxes (central line, and lower and upper border, respectively), and minimum and maximum by the T-bars (circles outliers, $>1.5$ times interquartile range; asterisks extreme outliers, $>3$ times interquartile range)

with an increase in toxicity grade included percent liver involvement $(P=0.038)$, baseline performance status $(P=$ $0.017)$, and prior liver resection $(P=0.020)$.

RE-induced liver disease (REILD), defined as liver failure, hyperbilirubinaemia and ascites in the absence of tumour progression, occurred in five patients $(4.5 \%)$. $D_{\mathrm{FL}-\mathrm{TOT}}$ was associated with REILD $(P=0.011)$. In addition, pretreatment AST $(P=0.006)$ and ALT $(P=0.035)$, performance status $(P=$ $0.038)$, and previous $\mathrm{RE}(P=0.031)$ were also associated with REILD. Interestingly, three of the five patients had received previous radiation therapy to the liver, either RE or external beam radiation therapy (EBRT). The median $D_{\text {FL-TOT }}$ in patients with REILD was $41.2 \mathrm{~Gy}$, compared to 27.9 Gy in the other patients $(P=0.011)$. However, corrected for previous radiation exposure calculated by dose-volume histogram (DVH) analysis [21], the cumulative $D_{\text {FL-TOT was }} 64.8 \mathrm{~Gy}$ versus $27.9 \mathrm{~Gy}(P=0.009)$. Five of 13 patients $(38.5 \%)$ with cumulative $D_{\mathrm{FL}-\mathrm{TOT}}>60$ Gy had REILD $(P<0.001)$. None had progressive disease during follow-up (partial response in three patients, stable disease in one patient, no imaging in one patient with biopsy-proven venoocclusive disease), but median survival in these five patients was only 84 days.

The calculated $D_{\mathrm{T}} / D_{\mathrm{FL}-\mathrm{TOT}}$ ratio, analogous to a physiological tumour to normal (T/N) ratio, was $<1$ in 41 of the 122 patients $(33.6 \%)$. This was encountered significantly more frequent in patients with secondary malignancy (33 of the 41 patients; $P=0.009$ ), often in patients with large overlap areas that were positive for both TcMAA and TcSC. In contrast to anatomical segmentation methods these overlapping areas were included in the normal liver segment, which leads to generally lower $\mathrm{T} / \mathrm{N}$ ratios. Efficacy and toxicity parameters 
were not found to be associated with $D_{\mathrm{T}} / D_{\mathrm{FL}-\mathrm{TOT}}<1$. $D_{\mathrm{T}} / D_{\mathrm{FL}}$ тот depended on tumour cell type (Table 2), but was not correlated with response at 3 and 6 months, toxicity, toxicity grade increase, or REILD.

Of the 122 patients, 24 were treated with glass microspheres (22 with HCC). Compared to patients treated with resin microspheres (4 of 98 with $\mathrm{HCC}$ ), patients treated with glass microspheres had a statistically significant worse baseline ECOG performance status and liver function with $75 \%$ known to have liver cirrhosis versus $4 \%(P<0.001)$. They were treated with higher activities, which resulted in higher $D_{\mathrm{T}}$ and $D_{\text {FL-TOT. }}$ Response rates at 3 months (64\% versus $35 \% ; P=0.069)$ and 6 months (78 \% versus $40 \% ; P=$ 0.064 ) showed a trend in favour of glass microspheres, but survival showed no difference (heavily biased by HCC). Response at 3 months was associated with prolonged survival in both groups (median 8.8 versus 39.1 months for resin, and 6.5 versus 19.8 months for glass; $P=0.005$ ). With regard to toxicity, worse baseline characteristics and higher $D_{\text {FL-TOT }}$ after treatment with glass microspheres did not result in any association between glass microspheres and increased toxicity, perhaps also because fewer patients received whole-liver treatment compared to those treated with resin microspheres ( $42 \%$ versus $89 \% ; P<0.001)$. Microsphere type (i.e. resin or glass) was included as a parameter in the multivariate analysis, but was not independently associated with efficacy or toxicity (see above). Liver cirrhosis was associated with worse baseline laboratory values, but did not result in increased toxicity per se (being associated with absolute toxicity grade, but not with changes in toxicity grade). As expected, patients with cirrhosis did have a worse survival than noncirrhotic patients (median 5.2 versus 10.8 months; $P=0.033$ ).

\section{Discussion}

Fusion TcMAA/TcSC SPECT imaging enables physiological partitioning of the liver for intrahepatic RE dosimetry. It allows calculation of the absorbed dose to the tumour and to the functional liver parenchyma, even in patients with extensive infiltrative and/or multifocal disease in whom anatomical imaging-based partitioning is not feasible [1]. Across a large variety of primary and metastatic liver tumours, we found that tumour dose $D_{\mathrm{T}}$ was correlated with objective response and overall survival, and functional liver dose $D_{\text {FL-TOT was corre- }}$ lated with toxicity. This study clearly confirmed dose-response relationships in a large heterogeneous cohort of patients typical of the population treated by RE.

Previous partitioning methods based on morphological anatomical imaging have been applied on patients with limited disease $[22,23]$. Garin et al. segmented the liver on TcMAA SPECT/CT images by semiautomatic generation of a volume of interest over the tumour on the SPECT images using an isocontour method to match the tumour on the CT images. In a preliminary report in $36 \mathrm{HCC}$ patients they showed that a threshold $D_{\mathrm{T}}$ value of $205 \mathrm{~Gy}$ was predictive of response [10]. These findings were confirmed in the extended cohort of 71 patients. Dosimetry enabled treatment intensification with favourable clinical outcome in selected patients, especially in patients with large tumours and portal vein thrombosis [24]. Mazzaferro et al. also found a correlation between $D_{\mathrm{T}}$ and response in $52 \mathrm{HCC}$ patients, albeit at a higher threshold value of 500 Gy using manual delineation of the tumour [25]. A maximum safety threshold for normal liver parenchyma of $70 \mathrm{~Gy}$ was advocated based on these data [26]. Although promising, anatomical partitioning is limited in clinical practice, mainly because the presence of extensive or diffuse malignancy is associated with substantial error. Also, these methods do not adequately account for the compartment that contains both tumour and functional liver directly surrounding the tumour.

In our study, $D_{\mathrm{T}}$ was also correlated with response and with survival. In addition, toxicity was associated with the functional liver dose $D_{\mathrm{FL}-\mathrm{TOT}}$, a finding consistent with existing understanding of radiation hepatotoxicity [27]. Patients with REILD had a significantly higher $D_{\text {FL-TOT, }}$ often due to previous hepatic radiation exposure. Prior EBRT, as well as prior $\mathrm{RE}$ treatment of the same target volume, is known to increase RE toxicity [21, 28]. When DVH analysis and voxel-based fusion SPECT dosimetry were applied to our cohort, we found that cumulative $D_{\text {FL-TOT }}$ was above 60 Gy in all patients with REILD. The probability of REILD was further increased by poor performance status and poor liver function at baseline.

The volume of the unirradiated part of the functional liver $\left(V_{\mathrm{FL}-U N}\right)$ proved to be associated with toxicity grade change and REILD, and with survival. This mirrors the surgical tenet that sufficient functional liver must be preserved for a better outcome after resection. A future liver remnant (FLR) fraction larger than $20-30 \%$ is recommended in patients with a normal liver, whereas a remnant of $>40 \%$ is recommended in patients with cirrhosis [29]. The surgical concept of FLR could be adapted for application to RE, with thresholds to be defined for both the minimum volume of and the maximum dose to 'unirradiated functional liver'.

Our ratio $D_{\mathrm{T}} / D_{\mathrm{FL}-\mathrm{TOT}}$ is distinctly different from the commonly cited $\mathrm{T} / \mathrm{N}$ ratio. Our physiological definition of functional liver assigns marginal tissue to the 'normal' compartment, which probably includes hypervascular rims and illdefined tumours interspersed with functional parenchyma. In contrast, the more commonly used $\mathrm{T} / \mathrm{N}$ ratio is based on anatomical segmentation, where ' $\mathrm{T}$ ' probably includes some functional liver surrounding the tumour. Assigning these irradiated areas to ' $\mathrm{T}$ ' will increase the $\mathrm{T} / \mathrm{N}$ ratio in comparison to our physiological $D_{\mathrm{T}} / D_{\mathrm{FL}-\mathrm{TO}}$. Including the overlap area in $D_{\mathrm{FL}-\mathrm{TOT}}$ resulted in more accurate prediction of toxicity as would be expected from the scintigraphic characteristics. Some investigators have advocated using the ratio to guide 
patient selection [30, 31], finding that the FDG PET response in $\mathrm{mCRC}$ lesions can be predicted using a cut-off $\mathrm{T} / \mathrm{N}$ ratio of 1 [30]. However, this remains controversial, since another study in $58 \mathrm{mCRC}$ patients found no correlation with response [32]. ' $\mathrm{T}$ ' and ' $\mathrm{N}$ ' compartments were defined by morphological imaging only, which may have contributed to the lack of correlation. Our method using physiological characterization showed that the ratio $D_{\mathrm{T}} / D_{\mathrm{FL}-\mathrm{TOT}}$ was not correlated with efficacy or toxicity; rather, the actual values of $D_{\mathrm{T}}$ and $D_{\mathrm{FL}-\mathrm{TOT}}$ were more predictive.

Based on the results of this study, some preliminary suggestions may be provided for prospective use of dual-tracer SPECT dosimetry. None of the patients with REILD had a $D_{\text {FL-TOT }}<30$ Gy from RE or a cumulative $D_{\text {FL-TOT }}<60$ Gy. This suggests an alternative strategy for dose prescription: to adjust the administered activity to keep $D_{\text {FL-TOт }}$ below a risk threshold. Surprisingly, a cumulative $D_{\mathrm{FL}-\mathrm{TOT}}$ of $30-60$ Gy appeared to be well tolerated, but the risk of REILD if $D_{\text {FL-TOT }}$ exceeded 60 Gy was $38 \%$. A $D_{\mathrm{T}}>32.7$ Gy led to a $50 \%$ objective response rate, irrespective of tumour cell type. It seems reasonable to aim for $D_{\mathrm{T}}>32.7$ Gy if cumulative $D_{\mathrm{FL}-}$ тот can be kept below $30 \mathrm{~Gy}$. Under this proposal, of the 122 patients in our cohort, administered activity could potentially have been increased in 65 patients $(53 \%)$. Thirteen patients (11\%), including the five with REILD, would have required activity reduction to keep $D_{\text {FL-TOT }}$ below 30 Gy. This strategy will need to be validated using a prospective protocol.

Threshold $D_{\mathrm{T}}$ and $D_{\text {FL-TOT }}$ may prove to be different between glass and resin microspheres. These products differ in activities prescribed, specific activity, embolization effect, and often proportion of the liver treated. Dose-effect relationships for both efficacy and toxicity are influenced by these differences. Dose-effect relationships probably also differ for each tumour cell type and for different baseline liver function statuses. With additional experience, thresholds should be defined tailored to different tumour cell types and product used. However, even with our amalgamated heterogeneous cohort, significant dose-response relationships were identified.

The limitations of this study include its retrospective design and imperfect toxicity and efficacy analysis. Strong endpoints, such as REILD, had a low incidence, while weaker endpoints, such as grade $3 / 4$ toxicity, were heavily confounded by pretreatment morbidity, tumour cell type, and disease progression. Response evaluation was confounded by the premature death of patients who were censored, resulting in biased response rates.

The reported absolute absorbed doses in tumorous and nontumorous tissue should be considered with care, since absolute SPECT quantification in the current study was prone to error due to the use of older technology. Although clinical SPECT can be quantitative with errors of less than $10 \%$, it requires careful set-up and calibration, as well as state-of-theart SPECT/CT systems and iterative reconstruction software able to accurately model the imaging physics, and to compensate for image-degrading factors (i.e. attenuation, scatter and partial volume) [33].

Work in progress includes optimization of image analysis using SPECT/CT and CT-based attenuation correction (not available at the time), dose-point kernel algorithms, and SPECT-based DVH analysis. Automatic threshold-based segmentation will be refined based on these techniques, to find a balance between the threshold used and the segmented volume. Future studies will focus on defining absorbed dose threshold values for fusion dual-tracer SPECT dosimetry in a prospective controlled setting.

\section{Conclusion}

Fusion TcMAA/TcSC SPECT imaging is a true physiologybased functional imaging tool that reveals dose-response relationships for hepatic RE. Absorbed doses in tumours and in functional liver tissue correlate with response, survival and toxicity in a heterogeneous population. This method may be useful for individualized treatment planning.

\section{Compliance with ethical standards}

Conflicts of interest Daniel Sze: Medical advisory boards for Surefire Medical, Treus Medical, RadGuard, Jennerex Biotherapeutics, Koli Medical, and Lunar Design; consultant for BTG, Sirtex, Covidien, and W. L. Gore. Marnix Lam: speaker for Sirtex; medical advisory board Bayer Healthcare; consultant for BTG. The other authors declare that they have no competing interests.

Authors' contributions M.L., M.G. and D.S. designed the study. M.L. and D.S. acquired and analysed the data and wrote the manuscript, and are accountable for all aspects of the work. A.B... acquired and analysed the data. A.I., E.M., J.L. and M.G. interpreted the data and revised the manuscript. All authors read and approved the final manuscript.

Statement of human rights All procedures performed in studies involving human participants were in accordance with the ethical standards of the institutional and/or national research committee and with the principles of the 1964 Declaration of Helsinki and its later amendments or comparable ethical standards. For this type of study formal consent is not required.

Statement on the welfare of animals This article does not describe any studies with animals performed by any of the authors.

Informed consent Data were handled in accordance with the Health Insurance Portability and Accountability Act. The institutional review board approved the study and waived the need for informed consent for this retrospective study.

Open Access This article is distributed under the terms of the Creative Commons Attribution 4.0 International License (http:// creativecommons.org/licenses/by/4.0/), which permits unrestricted use, distribution, and reproduction in any medium, provided you give appropriate credit to the original author(s) and the source, provide a link to the Creative Commons license, and indicate if changes were made. 


\section{References}

1. Lau WY, Kennedy AS, Kim YH, Lai HK, Lee RC, Leung TW, et al. Patient selection and activity planning guide for selective internal radiotherapy with yttrium-90 resin microspheres. Int J Radiat Oncol Biol Phys. 2012;82(1):401-7.

2. Gray BN, Burton MA, Kelleher DK, Anderson J, Klemp P. Selective internal radiation (SIR) therapy for treatment of liver metastases: measurement of response rate. J Surg Oncol. 1989;42(3): 192-6.

3. Gray B, Van Hazel G, Hope M, Burton M, Moroz P, Anderson J, et al. Randomised trial of SIR-Spheres plus chemotherapy vs. chemotherapy alone for treating patients with liver metastases from primary large bowel cancer. Ann Oncol. 2001;12(12):1711-20.

4. Gray BN, Anderson JE, Burton MA, van Hazel G, Codde J, Morgan C, et al. Regression of liver metastases following treatment with yttrium-90 microspheres. Aust N Z J Surg. 1992;62(2): 105-10.

5. Stubbs RS, Cannan RJ, Mitchell AW. Selective internal radiation therapy with 90yttrium microspheres for extensive colorectal liver metastases. J Gastrointest Surg. 2001;5(3):294-302.

6. Van Hazel G, Blackwell A, Anderson J, Price D, Moroz P, Bower $\mathrm{G}$, et al. Randomised phase 2 trial of SIR-Spheres plus fluorouracil/ leucovorin chemotherapy versus fluorouracil/leucovorin chemotherapy alone in advanced colorectal cancer. J Surg Oncol. 2004;88(2):78-85.

7. Andrews JC, Walker SC, Ackermann RJ, Cotton LA, Ensminger WD, Shapiro B. Hepatic radioembolization with yttrium-90 containing glass microspheres: preliminary results and clinical followup. J Nucl Med. 1994;35(10):1637-44.

8. Cao X, He N, Sun J, Tan J, Zhang C, Yang J, et al. Hepatic radioembolization with Yttrium-90 glass microspheres for treatment of primary liver cancer. Chin Med J (Engl). 1999;112(5): 430-2.

9. Kao YH, Tan EH, Ng CE, Goh SW. Clinical implications of the body surface area method versus partition model dosimetry for yttrium-90 radioembolization using resin microspheres: a technical review. Ann Nucl Med. 2011;25(7):455-61.

10. Garin E, Lenoir L, Rolland Y, Edeline J, Mesbah H, Laffont S, et al. Dosimetry based on $99 \mathrm{mTc}$-macroaggregated albumin SPECT/CT accurately predicts tumor response and survival in hepatocellular carcinoma patients treated with 90Y-loaded glass microspheres: preliminary results. J Nucl Med. 2012;53(2):255-63.

11. Kao YH, Hock Tan AE, Burgmans MC, Irani FG, Khoo LS, Gong Lo RH, et al. Image-guided personalized predictive dosimetry by artery-specific SPECT/CT partition modeling for safe and effective 90Y radioembolization. J Nucl Med. 2012;53(4):559-66.

12. SIR-Spheres microspheres (Yttrium-90 Microspheres) (Package Insert). North Sydney, Australia: Sirtex Medical; 2013.

13. Lam MG, Goris ML, Iagaru AH, Mittra ES, Louie JD, Sze DY. Prognostic utility of $90 \mathrm{Y}$ radioembolization dosimetry based on fusion 99mTc-macroaggregated albumin-99mTc-sulfur colloid SPECT. J Nucl Med. 2013;54(12):2055-61.

14. Coldwell D, Sangro B, Wasan H, Salem R, Kennedy A. General selection criteria of patients for radioembolization of liver tumors: an international working group report. Am J Clin Oncol. 2011;34(3):337-41.

15. Lewandowski RJ, Sato KT, Atassi B, Ryu RK, Nemcek Jr AA, Kulik L, et al. Radioembolization with 90Y microspheres: angiographic and technical considerations. Cardiovasc Intervent Radiol. 2007;30(4):571-92.

16. Kennedy A, Nag S, Salem R, Murthy R, McEwan AJ, Nutting C, et al. Recommendations for radioembolization of hepatic malignancies using yttrium-90 microsphere brachytherapy: a consensus panel report from the radioembolization brachytherapy oncology consortium. Int J Radiat Oncol Biol Phys. 2007;68(1):13-23.

17. TheraSphere Yttrium-90 Glass Microspheres (Package Insert). Ottawa: Nordion; 2013.

18. Lencioni R. New data supporting modified RECIST (mRECIST) for Hepatocellular Carcinoma. Clin Cancer Res. 2013;19(6): 1312-4.

19. Chalian H, Tore HG, Horowitz JM, Salem R, Miller FH, Yaghmai V. Radiologic assessment of response to therapy: comparison of RECIST Versions 1.1 and 1.0. Radiographics. 2011;31(7): 2093-105.

20. Gulec SA, Mesoloras G, Stabin M. Dosimetric techniques in 90Ymicrosphere therapy of liver cancer: the MIRD equations for dose calculations. J Nucl Med. 2006;47(7):1209-11.

21. Lam MG, Abdelmaksoud MH, Chang DT, Eclov NC, Chung MP, Koong AC, et al. Safety of $90 \mathrm{Y}$ radioembolization in patients who have undergone previous external beam radiation therapy. Int $\mathrm{J}$ Radiat Oncol Biol Phys. 2013;87(2):323-9.

22. Ho S, Lau WY, Leung TW, Chan M, Ngar YK, Johnson PJ, et al. Partition model for estimating radiation doses from yttrium-90 microspheres in treating hepatic tumours. Eur J Nucl Med. 1996;23(8):947-52.

23. Ho S, Lau WY, Leung TW, Chan M, Johnson PJ, Li AK. Clinical evaluation of the partition model for estimating radiation doses from yttrium-90 microspheres in the treatment of hepatic cancer. Eur J Nucl Med. 1997;24(3):293-8.

24. Garin E, Lenoir L, Edeline J, Laffont S, Mesbah H, Poree P, et al. Boosted selective internal radiation therapy with 90Y-loaded glass microspheres (B-SIRT) for hepatocellular carcinoma patients: a new personalized promising concept. Eur J Nucl Med Mol Imaging. 2013;40(7):1057-68.

25. Mazzaferro V, Sposito C, Bhoori S, Romito R, Chiesa C, Morosi C, et al. Yttrium-90 radioembolization for intermediate-advanced hepatocellular carcinoma: a phase 2 study. Hepatology. 2013;57(5): 1826-37.

26. Chiesa C, Mira M, Maccauro M, Romito R, Spreafico C, Sposito C, et al. A dosimetric treatment planning strategy in radioembolization of hepatocarcinoma with $90 \mathrm{Y}$ glass microspheres. Q J Nucl Med Mol Imaging. 2012;56(6):503-8.

27. Lawrence TS, Robertson JM, Anscher MS, Jirtle RL, Ensminger WD, Fajardo LF. Hepatic toxicity resulting from cancer treatment. Int J Radiat Oncol Biol Phys. 1995;31(5):1237-48.

28. Lam MG, Louie JD, Iagaru AH, Goris ML, Sze DY. Safety of repeated yttrium-90 radioembolization. Cardiovasc Intervent Radiol. 2013;36(5):1320-8.

29. Breitenstein S, Apestegui C, Petrowsky H, Clavien PA. "State of the art" in liver resection and living donor liver transplantation: a worldwide survey of 100 liver centers. World J Surg. 2009;33(4):797-803.

30. Flamen P, Vanderlinden B, Delatte P, Ghanem G, Ameye L, Van Den Eynde M, et al. Multimodality imaging can predict the metabolic response of unresectable colorectal liver metastases to radioembolization therapy with Yttrium-90 labeled resin microspheres. Phys Med Biol. 2008;53(22):6591-603.

31. Lambert B, Sturm E, Mertens J, Oltenfreiter R, Smeets P, Troisi R, et al. Intra-arterial treatment with $90 \mathrm{Y}$ microspheres for hepatocellular carcinoma: 4 years experience at the Ghent University Hospital. Eur J Nucl Med Mol Imaging. 2011;38(12):2117-24.

32. Dhabuwala A, Lamerton P, Stubbs RS. Relationship of 99mtechnetium labelled macroaggregated albumin $(99 \mathrm{mTc}-$ MAA) uptake by colorectal liver metastases to response following Selective Internal Radiation Therapy (SIRT). BMC Nucl Med. 2005;5:7.

33. Ritt $\mathrm{P}$, Vija H, Hornegger J, Kuwert T. Absolute quantification in SPECT. Eur J Nucl Med Mol Imaging. 2011;38 Suppl 1:S69-77. 